\title{
La ecografía clínica en el diagnóstico diferencial de las infecciones del aparato locomotor
}

\section{Cesar Henriquez*}

Instituto Ramón y Cajal de Investigación Sanitaria (IRICYS), Madrid-España *Correspondencia: chenriquezc@gmail.com

La ecografía, como método diagnóstico, se viene practicando desde la década de los 50, cuando se experimentó su uso en obstetricia. Sin embargo fue en la década de los 90, cuando los ecógrafos fueron tecnológicamente mejores, más fáciles de usar y más portátiles. Fué en esos años cuando se empezó a usar la ecografía por médicos no radiólogos como intensivitas, anestesiólogos, emergenciólogos, neumólogos, reumatólogos y más recientemente por internistas. [1, 2]

Los beneficios de usar la ecografía clínica superan con creces las limitaciones de esta herramienta diagnóstica. Tal es así, que cada vez es más frecuente realizar procedimientos guiados por ecografía como toracocentesis, paracentesis, pericardiocentesis, punciones lumbares, artrocentesis y drenajes de partes blandas, entre otros [3]. Tal es la diversidad de aplicaciones de la ecografía que resulta cada vez más factible que sea usado en diferentes escenarios clínicos [3].

Las infecciones de partes blandas son tratadas desde un punto de vista clínico. Sin embargo la ecografía clínica ha demostrado su utilidad en el manejo de pacientes con infecciones de partes blandas como celulitis, abscesos o fascitis $[4,5,6]$. Por ejemplo, la ecografía clínica puede determinar la extensión de una celulitis y sus complicaciones como la formación de abscesos o la extensión a planos profundos [7]. La imagen de una celulitis varía desde un edema difuso con una mayor ecogenicidad en la piel y el tejido celular subcutáneo hasta una imagen en empedrado que se traduce en liquido perifascial [8]. Si bien esta última no es específica de celulitis, es de gran utilidad correlacionar los signos y síntomas clínicos con la imagen ecográfica. En cuanto a la piomiositis, la apariencia ecográfica revela un edema muscular difuso, como si se tratara de una imagen en negativo de la apariencia ecográfica normal del músculo [8, 9]. Finalmente los hallazgos ecográficos de una fascitis necrosante varían de acuerdo al tiempo de evolución de la enfermedad y van desde el engrosamiento difuso de la fascia con capas de liquido hasta una apariencia de sombras sucias indicando la presencia de gas dentro del área de inflamación [10].

También la ecografía nos ayuda a determinar si un aumento de volumen articular se debe a compromiso de partes blandas o compromiso articular o de bursas. El liquido articular de una artritis séptica generalmente es hipoecoico con lo que claramente se diferencia de la sinovia articular y la cápsula articular. El derrame sinovial origina una imagen hipo o anecoica en los recesos sinoviales que desplaza la cápsula articular. La hipertrofia de la sinovia se objetiva como una banda irregular hipoecogénica en relación con los tejidos circundantes pero hiperecogénica en relación al derrame. También es posible visualizar líquido alrededor de los tendones como es el caso de la tenosinovitis infecciosa [11]. Dado que podemos visualizar el derrame en tiempo real a través de los recesos articulares, podemos realizar una punción guiada por ecografía de los derrames sinoviales y así obtener una muestra para estudios citológicos, bioquímicos y microbiológicos [12].

Por todo ello, es fundamental la enseñanza de la ecografía para el mejor abordaje de infecciones de partes blandas. Se han realizado estudios en estudiantes en quienes se consigue alcanzar un alta sensibilidad diagnostica tras un entrenamiento en ecografía $[13,14]$. En la mayor parte de los programas de residencia de reumatología en España, se contempla la enseñanza de la ecografía a pie de cama como una herramienta de ayuda diagnóstica y para las punciones y aspiraciones de liquido sinovial. El desafío para los próximos años es conseguir una mayor difusión de la enseñanza de la ecografía en otras especialidades que tratan pacientes con infecciones de partes blandas como infectología, medicina interna, medicina de familia y urgencias entre otras. 


\section{Referencias}

1. Moore CL, Copel JA. Point-of-care ultrasonography. N Engl J Med. 2011 Feb 24; 364(8):749-57.

2. Beltrán LM, García-Casasola G, members of the Group of Clinical Ultrasound of the Spanish Society of Internal Medicine. Ultrasonography managed by internists: the stethoscope of 21st century? Rev Clin Esp. 2014 Apr; 214(3):155-60.

3. Henriquez-Camacho C, Garcia-Casasola G, Guillén-Astete C, Losa J. Ultrasound for the diagnosis of infectious diseases: Approach to the patient at point of care and at secondary level. J Infect. 2015 Jul; 71(1):1-8.

4. Alsaawi A, Alrajhi K, Alshehri A, Ababtain A, Alsolamy S. Ultrasonography for the diagnosis of patients with clinically suspected skin and soft tissue infections: a systematic review of the literature. Eur J Emerg Med Off J Eur Soc Emerg Med. 2017 Jun; 24(3):162-9.

5. Barbic D, Chenkin J, Cho DD, Jelic T, Scheuermeyer FX. In patients presenting to the emergency department with skin and soft tissue infections what is the diagnostic accuracy of pointof-care ultrasonography for the diagnosis of abscess compared to the current standard of care? A systematic review and metaanalysis. BMJ Open. 2017 Jan 10; 7(1):e013688.

6. Subramaniam S, Bober J, Chao J, Zehtabchi S. Point-of-care Ultrasound for Diagnosis of Abscess in Skin and Soft Tissue Infections. Acad Emerg Med Off J Soc Acad Emerg Med. 2016 Nov; 23(11):1298-306.

7. Lewis DL, Butts CJ, Moreno-Walton L. Facing the danger zone: the use of ultrasound to distinguish cellulitis from abscess in facial infections. Case Rep Emerg Med. 2014; 2014:935283.

8. Chau CLF, Griffith JF. Musculoskeletal infections: ultrasound appearances. Clin Radiol. 2005 Feb; 60(2):149-59.

9. Kumar MP, Seif D, Perera P, Mailhot T. Point-of-Care Ultrasound in Diagnosing Pyomyositis: A Report of Three Cases. J Emerg Med. 2014 Apr 8;

10. Torrejón MDC, Celi E, Cancho D, Knox A, Henriquez-Camacho C. Necrotizing myositis in a neutropenic patient: the use of ultrasound in the diagnosis of atypical presentations. Case Rep Emerg Med. 2014; 2014:685263.
11. Guillén Astete C, Rodríguez García A, Prieto Morales M, Zea Mendoza A. Infectious tenosynovitis of the foot extensors. Reumatol Clin. 2014 Feb; 10(1):56-7.

12. Costantino TG, Roemer B, Leber EH. Septic arthritis and bursitis: emergency ultrasound can facilitate diagnosis. J Emerg Med. 2007 Apr; 32(3):295-7.

13. Galusko V, Khanji MY, Bodger O, Weston C, Chambers J, Ionescu A. Hand-held Ultrasound Scanners in Medical Education: A Systematic Review. J Cardiovasc Ultrasound. 2017 Sep; 25(3):7583.

14. Garcia-Casasola G, Sánchez FJG, Luordo D, Zapata DF, Frías MC, Garrido VV, et al. Basic Abdominal Point-of-Care Ultrasound Training in the Undergraduate. J Ultrasound Med. 2016 Nov 1; 35(11):2483-9.

\section{Publique en Acta Reumatológica}

Acta Reumatológica es una revista que tiene por fin la difusión de estudios clínicos relacionados con aspectos prácticos del diagnóstico, tratamiento y seguimiento de pacientes con patología reumatológica, de estudios epidemiológicos relacionados con patología inflamatoria y musculoesquelética de presentación común o infrecuente en la práctica clínica tanto en población adulta como pediátrica, de casos clínicos de patología poco habitual o de presentaciones inhabituales de patología frecuente, de imágenes didácticas e ilustrativas en reumatología y del estado actual e innovación en la formación especializada en reumatología. 